\title{
Análisis de los incidentes críticos de la identidad profesional docente del profesorado principiante de educación especial
}

\author{
Maite Otondo-Briceño ${ }^{{ }^{\star}}$, Cristina Mayor-Ruiz ${ }^{2}$ y Elena Hernández-de la Torre ${ }^{2}$ \\ (1) Universidad Católica de la Santísima Concepción, Chile, Departamento de Fundamentos Pedagógicos, Facultad de \\ Educación, Alonso de Ribera 2850, Concepción, Chile (correo-e: maite@ucsc.cl) \\ (2) Departamento de Didáctica y Organización Educativa, Facultad de Educación, Universidad de Sevilla, \\ C/ Pirotecnia s/n, Sevilla, España. (correo-e: crismayr@us.es; eht@us.es)
}

Recibido Dic. 10, 2020; Aceptado Feb. 9, 2021; Versión final Abr. 12, 2021, Publicado Ago. 2021

\begin{abstract}
Resumen
Esta investigación describe episodios que condicionan las trayectorias individuales y profesionales del profesorado principiante de educación especial titulados en una universidad chilena, a través de la técnica de análisis de incidentes críticos. La identidad docente es uno de los cimientos esenciales en la formación inicial y continua del profesorado. Este estudio es de corte biográfico-narrativo con una muestra de 23 profesoras principiantes, donde se aplica una entrevista semi-estructurada. Los resultados muestran incidentes críticos prototípicos con estrategias de resolución débilmente eficientes que interpelan a la formación y permiten entender los cambios producidos en las trayectorias laborales que han ayudado a tomar nuevos rumbos personales y profesionales. Se concluye que comprender qué aspectos han desarrollado la identidad profesional docente proporciona elementos que a través de su análisis y aplicación son una caja de resonancia para la formación futura del profesorado de educación especial, enriqueciendo de manera recíproca la teoría y práctica.
\end{abstract}

\section{Analysis of critical incidents in the professional teaching identity of novice special education teachers}

\begin{abstract}
This research study describes episodes that condition the professional careers of novice special education teachers graduated from a Chilean university by using the critical incident analysis technique. Teaching identity is an essential concept in the initial and continuous training of teachers. The present study applies a biographical-narrative with a sample of 23 novice teachers. A semi-structured interview is applied. The results show that prototypical critical incidents are identified by weakly efficient resolution strategies that challenge teacher training. These incidents allow understanding the changes that teachers experience throughout their professional careers and that has guided them to take new personal and professional directions. It is concluded that understanding what aspects develop professional teaching identity provides elements that, through their analysis and application, can guide future training of special education teachers by enriching teaching theory and practice.
\end{abstract}

Keywords: teacher professional identity; critical incidents; special education; novice 


\section{INTRODUCCIÓN}

Investigar sobre identidad o identidades profesionales docentes es complejo, no se puede reducir solo a analizar algunas dimensiones de ella, esto llevaría a una revisión reduccionista del problema (Cantón y Tardif, 2018; Bukor, 2015; Noonan, 2019). La identidad profesional docente (IPD) no es diferente a la identidad de otras profesiones $u$ otros aspectos que describen los mundos laborales, tales como: estatus social; roles y funciones que deben realizar; el lugar e importancia que cumplen en las organizaciones. La IPD no hace referencia a una "realidad empírica objetiva" (Cantón y Tardif, 2018, p.9), es una construcción teórica proveniente de variables psicosociales y formativas que dependen de marcos conceptuales y disciplinares diversos.

La IPD es la forma como el profesorado se define a sí mismo y también cómo definen a los otros. Tendría una construcción individual y otra colectiva (Jara y Mayor, 2019). Sería un conjunto heterogéneo de representaciones profesionales que responden a la diferenciación o identificación con otros grupos profesionales que se extiende desde y hacia sus experiencias personales y sociales en contextos específicos de desarrollo. La relevancia que tiene esta investigación en el contexto chileno, es que la IPD del profesorado de Educación Especial se encuentra en una encrucijada, puesto que los egresados de éstas titulaciones provienen de instituciones cuya Formación Inicial Docente (FID) está enfocada en la inclusión educativa, ingresan al mundo laboral con un sistema educacional que está transitando, con variadas tensiones, a un paradigma inclusivo, pero ahí se encuentran con una realidad laboral cuya práctica es un paradigma clínico, por lo que el docente principiante convive laboralmente con ambos paradigmas y en una permanente complejidad identitaria.

Es por ello que, la construcción de identidades debe volverse a la propia identidad, ya que las existentes, por cierto cambiantes, son facilitadoras o obstaculizadoras de las identidades a construir. Coincidente con lo planteado por los autores Albores y Avendaño (2016) la identidad evoluciona, tanto personal, como colectivamente, basada en la idea que el profesorado tiene de su profesión, y que en ocasiones es contraria a lo que encuentra en la realidad laboral (Correa et al., 2014). Es aquí donde se producen las contradicciones con relación a la IPD adoptada por parte del profesorado principiante, ya que, por un lado está el proceso formativo, por otro, está el marco normativo que regula el ejercicio profesional, están sus pares en ejercicio y el entorno social en su conjunto, la escuela, su historia y requerimientos, así que todo este entramado se relaciona con su desarrollo.

En consecuencia y de acuerdo a los antecedentes presentados, se vislumbra la necesidad de precisar la existencia de un vacío de conocimiento, se señala lo siguiente: (i) Aunque contamos con información acerca de los roles y funciones del profesorado de Educación Especial y de la IPD del profesorado en general, en Chile no se ha indagado suficientemente sobre qué factores o sucesos desarrollan la IDP de este profesional donde existen unas condiciones contextuales e institucionales que pueden ser determinantes para este fin; (ii) Conforme a las políticas públicas en esta área, concretamente en los Estándares Orientadores para titulaciones de Educación Especial en Chile (2013) para este sector, es importante estudiar qué ocurre con el desarrollo de la IPD de éste profesorado principiante; y (iii) Por último, para que el profesorado principiante de las Titulaciones de Pedagogía en Educación Especial contribuya al desarrollo de la calidad de la educación en el sistema educativo, es necesario brindar ciertos espacios formativos que permitan analizar sobre su IPD y qué factores, sucesos y eventos la van configurando y cambiando a través de todo el ejercicio profesional, es decir qué situaciones están implicadas en este proceso. El profesorado debe reflexionar sobre su propia IPD y darle un significado mediante los sucesos y cambios que esta experimenta. Una forma de llevarlo a cabo es a través de los incidentes críticos. Es decir, describir los episodios que han condicionado las trayectorias individuales y profesionales del profesorado principiante de Educación Especial de una Universidad chilena a través de ésta técnica de análisis.

Dado lo anterior, surge la necesidad de conocer qué factores acontecen en estas transformaciones identitarias docentes en el profesorado para contribuir en la mejora en la formación del profesorado y consecuentemente en la calidad educativa.

Las investigaciones que se realizan sobre IPD son abordadas desde distintas perspectivas, autores que se refieren a esto Marcelo y Vaillant (2015); Mulone (2016). Como característica común indican que es dinámica (Madueño y Márquez, 2020) se desarrolla con el tiempo y depende de variados factores como son el propio profesorado y el contexto social (Colliander, 2018). Cuando se hace referencia a lo dinámico, se quiere decir que no es fija o estática y que las transformaciones de ella se caracterizan por ser personales, relacionadas con trayectorias y decisiones propias y que dependen de los contextos. Esto significa que los docentes se identifican de distintas maneras según los lugares de trabajo o de formación, ya que, por diferentes razones, tienden a identificarse e involucrarse más con determinados contextos y responden de manera distinta a los requerimientos que estas comunidades les hacen (Colliander, 2018). Desde una perspectiva contemporánea, 
la IPD del profesorado se considera múltiple, discontinua, social, imbuida en mundos sociales donde las interdependencias causan cambios en el tiempo y en el entorno (Pappa et al., 2017).

\section{OTROS ANTECEDENTES}

Relacionado con lo social de la identidad, las características del profesorado evidencian los aspectos del desarrollo docente que cambian en coherencia con las interacciones y vinculaciones con las escuelas $u$ otros lugares donde desarrollan su profesión, acorde a la cultura escolar de esa comunidad y las políticas que allí están instaladas. El profesorado utiliza activamente las identidades que ha adquirido con anterioridad para aprender de esas comunidades, revisar prácticas de aula y adaptarse a las condiciones generales de la escuela (Colliander, 2018). En este sentido, Buchanan (2015) plantea cómo el profesorado recurre a sus identidades profesionales, que existen de manera previa en ellos, para poder dar una interpretación a las nuevas normas y responder a ellas.

Siguiendo esta línea argumentativa, la IPD que ha adquirido en la FID se constituyen en una parte fundamental que les permite situarse en las nuevas responsabilidades laborales que les corresponde asumir cuando se insertan en la profesión (Madueño y Márquez, 2020). La FID, vista como la formación inicial del profesorado, se constituye en un foco de preocupación para los responsables de esta formación por los grandes desafíos y demandas. Las solicitudes que se le hacen hoy a la educación tensionan el rol y las funciones del profesorado, quienes transitan desde la preocupación por la enseñanza, a formar a un estudiantado competente que posibilite el desarrollo del país. Esto afecta al desarrollo y construcción de la identidad de este grupo de profesionales (Madueño y Márquez, 2020).

La IPD, con relación a lo personal, según Rodgers y Scott (2008), viene a referir el significado que el profesorado tiene de sí mismo con relación a lo laboral, es decir, el concepto sobre su persona y de lo que hace en el ejercicio profesional, así como los quehaceres que debe realizar día a día, tales como: preparar clases, impartir clases, conocer y relacionarse con el estudiantado y con sus pares y relacionarse con sus superiores. También, señala qué elementos como conocimientos, creencias, emociones y actitudes, son los que dan forma a la identidad. Todo esto tiene relación en las decisiones que toman a través de su trayectoria profesional.

Considerando los planteamientos anteriores, la IPD, se desarrolla a partir de variados componentes. EI profesorado tiene una formación, la actualiza, imparte clases y forma parte de una institución educativa con normas y reglas, tiene muchas relaciones con el alumnado, con pares y padres de familia, y todo esto está vinculado con el desarrollo de su IPD. Asimismo, adapta sus prácticas a los requerimientos de las reformas de turno (Balderas, 2014) quien refiere que para comprender la IPD lo primero es señalar qué es lo que entendemos por identidad personal, social y laboral, ya que esto configura el entramado de lo que se entiende por ella. De esta manera Balderas (2014) se refiere a este concepto: La Identidad personal posibilita al sujeto la comprensión de sí mismo y de los otros, mediante el reconocimiento de semejanzas y desigualdades, contribuyendo en la adquisición de saberes, representaciones y subjetividades de la persona, se relaciona con el conocimiento sobre las cosas y las acciones realizadas; a través de este bagaje el individuo establece relaciones, decide a qué grupos sociales integrarse y es la base para la identidad social.

Con relación a la evolución de la identidad, esto acontece en la esfera de lo intersubjetivo y se define como un procedimiento evolutivo, de significación de uno mismo perteneciente a un contexto. Considerando esto, la IPD, da respuesta a preguntas como: ¿quién soy en este momento? De esta manera, no es una entidad estable o fija. Es decir, como señala Beijaard et al. (2004); Pillen et al. (2013), no es estática, sino que se va construyendo y modificando de acuerdo con la interpretación y reinterpretación que cada profesor/a hace de las experiencias vividas. Lo anterior, tiene relación con lo que hemos venido planteando, es decir, el carácter discontinuo y cambiante de la IPD. Correa et al. (2014) señala que su construcción es un proceso individual y colectivo que se inicia en la FID y se prolonga durante todo el ejercicio profesional, a través de las prácticas pedagógicas y en los primeros años de ejercicio profesional, se desarrolla a través de procesos de aprendizaje y afianzamiento (Madueño y Márquez, 2020).

Ciertamente, en la primera inserción laboral o primer trabajo, el profesorado principiante pone a prueba su creencia personal y vocacional, sus conocimientos previos, lo aprendido anteriormente en la formación y otros espacios de aprendizajes, lo rehace o lo cambia por nuevo aprendizaje. También, puede cambiar algunas habilidades iniciales, la sensación de efectividad, lo que cree y cómo actúa frente a ello (Correa et al., 2014), "estos autores señalan que las identidades docentes no sólo se forman a partir de teorías y contenidos relacionados con su profesión, sino que también se apoyan fuertemente en percepciones, interpretaciones y conocimientos que abarcan su ámbito personal y subjetivo y tienen una influencia significativa en su práctica profesional (p.449)" 
La IPD implica las explicaciones del sí mismo, del sujeto o del yo, con respecto a su identidad, como también la opinión de los otros con respecto al sujeto, "al identificarse con un conjunto de otros sujetos que pertenecen a una determinada ocupación o profesión, una persona establece una referencia no sólo a lo qué hace, sino también a quién es" (Mulone, 2016, p.154). De igual forma, Marcelo y Vaillant (2015) plantean que la IPD involucra muchos aspectos, es amplio, se adquiere cuando una persona se dedica y especializa profesionalmente en una tarea o en un área determinada, empieza con la formación inicial de las personas y continúa a lo largo de su vida profesional. Se construiría a través de un proceso y sería sostenido en el tiempo, resultaría de la combinación entre una parte que es común a todos los profesionales de esa área y también a una parte específica que estaría ligada a los contextos de trabajo. También, hace referencia a los significados que se atribuyen al itinerario o trayectorias de vivencias profesionales y va más allá de las fronteras de una determinada profesión u ocupación (Marcelo y Vaillant, 2015).

En los itinerarios y trayectorias profesionales, el profesorado pasa por diversas etapas formativas, tales como: FID, inserción laboral y formación continua. Las primeras experiencias laborales son importantes y la forma cómo las asumen estos profesionales; tienen un significado en los procesos de frustración - desilusión o en el desarrollo de actitudes de adaptación profesional. El profesorado principiante tiene tareas enseñar $y$ aprender a enseñar. A estas se agregan otras como conocer al estudiantado, el currículo y el contexto escolar; planear el currículo y la enseñanza; diseñar una colectividad de aprendizaje en el aula, y continuar desarrollando una IPD. El problema es cuando se le solicitan tareas y responsabilidades similares a las del profesorado con más experiencia, y es entonces cuando se presenta "un periodo de tensiones y aprendizajes intensivos en contextos generalmente desconocidos y durante el cual los docentes principiantes deben construir conocimiento profesional además de conseguir mantener un cierto equilibrio personal" (Marcelo et al., 2016, p.146). En esta etapa comienzan las primeras experiencias socializadoras de la profesión, por lo que no solo será importante aprender a ser un buen docente, sino a compartir experiencias, prácticas de enseñanza y la cultura escolar; en este periodo de inserción laboral esta socialización se produce con mayor intensidad. El profesorado principiante aprende a interiorizar las normas, valores, conductas, que caracterizan a la cultura escolar y profesional en la que se integra.

En el proceso de aprender a alcanzar estos elementos de forma espontánea se pueden desarrollar modelos de enseñanza que se contradicen con lo que solicitan las nuevas generaciones o la sociedad actual, y, para lograr mejores resultados en la etapa de adaptación y mejora en la inserción del profesorado, se necesita que, se promueva e impulse la indagación, innovación, reflexión y el crecimiento personal y profesional. Según Ávalos (2016) en una investigación sobre profesorado principiante, encontró que, la mayoría de los estudios realizados sobre este profesorado están referidos a: primero, procesos de inducción con relación a: descripción de programas, procesos y efectos; segundo, las referidas a enseñanza aprendizaje con relación a: aspectos cognitivos, afectivos, sociales y prácticos y tercero, el aprendizaje profesional reflexión, habilidades cognitivas, liderazgo.

Muchos de los problemas de los docentes principiantes tienen que ver con situaciones que enfrentan o han enfrentado docentes con mayor experiencia, tales como: la disciplina en el aula, la motivación del estudiantado, la organización del trabajo en clase, los materiales insuficientes, los problemas personales del estudiantado o las relaciones con los padres. Los maestros principiantes experimentan los problemas con mayores dosis de incertidumbre y estrés debido a que tienen menores referentes y mecanismos para enfrentar estas situaciones (Vaillant et al., 2016). En síntesis, es necesario reconocer que la inserción a la docencia no es un camino fácil de realizar, este se desarrolla en relación a otros, en un contexto con características propias, a una cultura institucional y una realidad educativa diversa. Por lo tanto, para que esta transición no esté rodeada de dificultades que provoque inseguridades e incertidumbres se sugiere un proceso de acompañamiento e inducción al mundo laboral.

\section{METODOLOGÍA}

La metodología de investigación adoptada ha sido de corte cualitativa utilizando el método biográfico narrativo (Bolívar et al., 2001). Se han considerado estudios de casos cruzados y entrevistas semiestructuradas para la recogida de datos (Pujadas, 2002); asimismo se ha optado por la entrevista biográfico narrativa (Pujadas, 2002) orientada temáticamente por un guión con una estructura flexible, con un tiempo de duración entre 60 a 90 minutos, grabadas y posteriormente transcritas. Los incidentes críticos se han analizado y caracterizado valorando sucesos positivos o negativos en la trayectoria de vida de las informantes. Los elementos consignados en el análisis de incidentes críticos han sido: título, tipo de incidente, sucesos y valoración según lo planteado por Bolívar et al. (2001).

La técnica de análisis de incidentes críticos es utilizada desde hace tiempo sobre todo en formación inicial del profesorado (Bolívar et al., 2001). El propósito que ha tenido su utilización, en este estudio, es que se pueda llegar a la comprensión de los sucesos que caracterizan la IPD del profesorado principiante desde su propio 
punto de vista (Deng et al.,2018). Esta técnica se centra en la experiencia subjetiva y permite analizar, cuantificar y categorizar las conductas. Considerando a Bolívar et al. (2001) "Ios incidentes críticos manifiestan aquellos eventos en la vida individual, seleccionados en función de que marcaron particulares direcciones o rumbos" (p.172). En el desarrollo de este estudio se han empleado situaciones que tienen implicaciones en la IPD.

\section{Diseño de la investigación}

Se ha abordado una metodología cualitativa, donde se muestra el análisis de los episodios que han condicionado las trayectorias individuales y profesionales del profesorado principiante de Educación Especial titulados de una Universidad chilena a través de la técnica de análisis de incidentes críticos (Bolívar et al., 2001) para comprender el desarrollo de la IPD de este profesorado. En la figura 1 se puede visualizar una gráfica donde emergieron los principales incidentes críticos: experiencias personales, formativas y laborales.

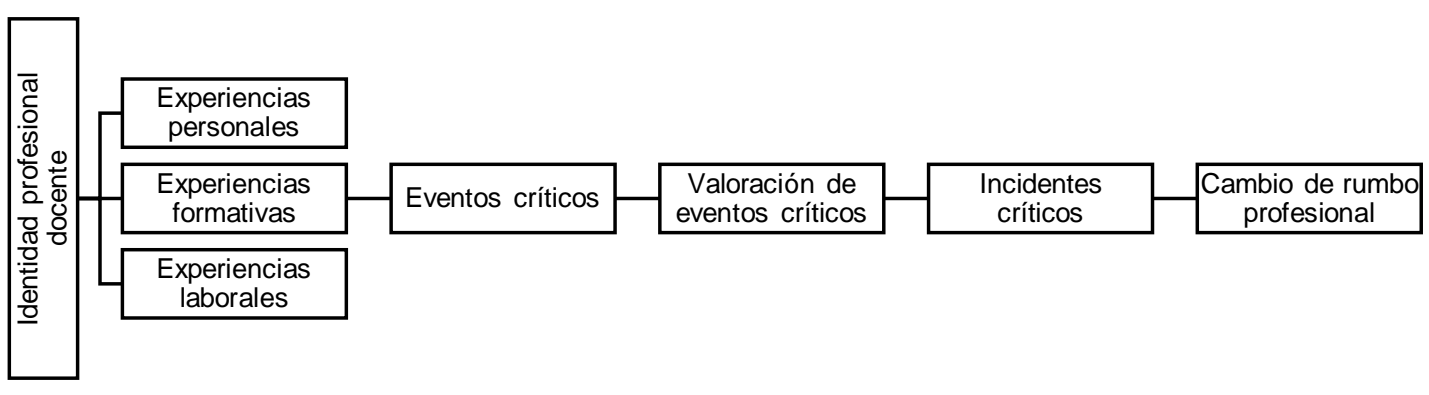

Fig. 1: Proceso análisis de incidentes críticos

Una vez revisada la literatura se han considerado los aportes de varios autores con el objeto de determinar formas y procedimientos de analizar la construcción de la IPD, es decir, la identidad docente está vinculada a las experiencias educativas y formativas personales y colectivas, pero también son situadas o determinadas por las experiencias sociales y personales. De esta manera, se determinan las dimensiones, categorías y subcategorías de la entrevista semiestructurada que fueron aplicadas al profesorado principiante y posteriormente transcritas para su análisis. Se ha realizado un análisis de contenido (Pujadas, 2002) enfocado en rescatar los momentos críticos experimentados por los docentes, cómo fueron valorados estos eventos y lo que ocurrió posteriormente en sus vidas como cambio de rumbo profesional (Bolívar et al., 2001).

\section{Muestra de la investigación}

La muestra se compone de profesorado principiante de Educación Especial según los siguientes criterios de inclusión: 1) profesorado con menos de 5 años de ejercicio profesional tituladas de la Universidad donde se realiza el estudio; 2) situación profesional estable en el área de Educación Especial desde el año 2013 al 2018; 3) profesorado dispuesto a colaborar. En total, fueron entrevistadas 23 profesoras procedentes de distintos establecimientos de la región del Bio Bio, Chile. En esta investigación se ha optado como definición de profesorado principiante aquel que se inicia en la profesión hasta 5 años de desarrollo en ella (Marcelo et al., 2016).

\section{Técnicas de recogida de información}

Se ha utilizado la entrevista semiestructurada validada por 9 expertos, 3 de ellos pertenecientes al Claustro de Doctorado en Consorcio de la Universidad implicada en el estudio, además 3 profesores de Educación Especial en ejercicio y 3 académicos pertenecientes a la planta docente de la titulación de Educación Especial de la Universidad. La estructura de la entrevista está compuesta por 4 dimensiones: Inicio, testimonio, desarrollo y final; 6 categorías y 28 subcategorías. En la Tabla 1 se presenta la estructura de la entrevista. Las entrevistas se han realizado de manera individual y se ha solicitado que respondieran preguntas relacionadas con las categorías de análisis apriorísticas, previamente propuestas, tales como: identificación, experiencias educativas formativas (antes de ingresar a la Universidad), experiencias educativas en la formación inicial, experiencia laboral, vivencias significativas e IPD. Posteriormente, se les ha solicitado que recordaran momentos críticos vividos que hayan sido significativos para ellas y de qué manera valoraban esas vivencias. Posteriormente, se les ha preguntado si esas vivencias habían tenido un cambio significativo en sus vidas. En la Tabla 1 se presenta la estructura de la entrevista semi estructurada, es decir: dimensiones, categorías y subcategorías. 
Tabla 1: Estructura de entrevista semi estructurada

\begin{tabular}{|c|c|c|}
\hline Dimensiones & Categoría & Subcategoría \\
\hline Inicio & Identificación & Auto presentación \\
\hline \multirow[t]{3}{*}{ Testimonio } & $\begin{array}{l}\text { Experiencias educativas (antes } \\
\text { de ingresar a la Universidad) }\end{array}$ & $\begin{array}{l}\text { Experiencias educativas } \\
\text { Profesores y características. } \\
\text { Sentimientos provocados. } \\
\text { Experiencias vocacionales } \\
\text { Acceso a la Universidad }\end{array}$ \\
\hline & $\begin{array}{l}\text { Experiencias educativas en } \\
\text { formación inicial }\end{array}$ & $\begin{array}{l}\text { Experiencias Universidad } \\
\text { Formación inicial docente } \\
\text { Descripción de la Universidad } \\
\text { Descripción de profesores } \\
\text { Características de profesores: afectivas, personales, académicas } \\
\text { Vivencias en la formación. } \\
\text { Sentimientos que le provocan. } \\
\text { Prácticas educativas. } \\
\text { Experiencias vocacionales }\end{array}$ \\
\hline & Experiencia laboral & $\begin{array}{l}\text { Experiencias laborales } \\
\text { Desempeño laboral } \\
\text { Valoración laboral } \\
\text { Ejercicio profesional } \\
\text { Expectativas laborales } \\
\text { Reforma educativa } \\
\text { Práctica docente } \\
\text { Participación en proyectos }\end{array}$ \\
\hline Desarrollo & Vivencias significativas & $\begin{array}{l}\text { Momentos críticos. } \\
\text { Autoimagen profesional } \\
\text { Vivencias con reformas educativas. } \\
\text { Relaciones cotidianas en su trabajo (alumnos, docentes, otros) } \\
\text { Nivel de bienestar laboral. } \\
\text { Fortalezas y debilidades, etc. }\end{array}$ \\
\hline Final & Identidad Profesional docente & Definición/Significado \\
\hline
\end{tabular}

\section{Obtención y análisis de datos}

Para la obtención y recogida de datos, como se ha señalado anteriormente, se ha entrevistado a 23 profesoras principiantes de Educación Especial con menos de 5 años de ejercicio de la profesión. El interés ha sido comprender el desarrollo de la IPD, siendo esencial que las participantes contaran sus experiencias y vivencias que han sido el foco de interés del trabajo. Las entrevistas han sido grabadas, registradas cada uno de los audios y posteriormente transcritas para facilitar el análisis que se describe a continuación considerando el modelo de Vloet et al. (2020): (1) en primer lugar, se han elegido trozos de texto vinculados a momentos, eventos conflictivos o incidentes críticos; (2) en segundo lugar, se ha diseñado un patrón para analizar los trozos elegidos de los momentos difíciles con relación a: el momento, el punto álgido, el enfoque dado por el profesorado en el momento del incidente, el efecto que produjo; (3) en tercer lugar, se ha considerado: el ordenamiento; la clasificación y componentes de la IPD; las valoraciones de estos incidentes; las situaciones importantes en la trayectoria de vida de estas profesoras y que han dejado un aprendizaje; (4) en cuarto lugar, se han clasificado los incidentes críticos, constatando la concurrencia de ello y su densidad; (5) en quinto lugar, se ha procedido a la identificación y categorización de cada tipo de incidente seleccionado, haciendo referencia a elementos importantes relacionados con la IPD. Cada uno de los incidentes críticos han sido asociado a las dimensiones, categorías y subcategorías con relación a la recurrencia y valoración entregadas por los informantes claves.

\section{RESULTADOS}

A continuación, presentamos los resultados, vinculándolos a las dimensiones y categorías apriorísticas de la investigación: dimensión 1-inicio; dimensión 2-testimonio; dimensión 3-desarrollo; y dimensión 4-final: IPD. 


\section{Dimensión 1. Inicio. Categoría: Identificación}

El profesorado principiante se identifica como profesorado de Pedagogía en Educación Especial y con la mención que desarrollaron durante la FID. En la Tabla 2 se puede observar los incidentes críticos asociados a esta dimensión y categoría.

Tabla 2: Incidentes críticos de dimensión Inicio

\begin{tabular}{|l|l|}
\hline Categoría & Incidentes críticos \\
\hline Identificación & Identificación con la Universidad de la formación inicial \\
\cline { 2 - 2 } & Primera generación acceso a la Universidad \\
\hline
\end{tabular}

Lo que llama la atención, en esta dimensión, son dos incidentes críticos. Primero, la importancia y valoración que le asignan a la identificación con la Universidad como entidad formadora, como estudiantado y ahora como profesionales. Esto ha sido relevante para ellas por la evaluación externa que se tiene de las profesionales egresadas de esta institución, lo que ha permitido encontrar trabajo antes de estar tituladas. Segundo, es ser la primera generación de su familia que tiene acceso a la Universidad, fue estimado como un hecho muy significativo, ya que cambia el rumbo en la toma de decisiones futuras, esta razón hace que, a pesar de no optar en primera instancia por esta titulación, permanezcan en ella y culminen su itinerario formativo. (.....) soy profesionalmente educadora diferencial mención Dificultades de Aprendizaje de la UCSC y especialista en Neurociencia aplicada a la educación". (Caso 1) y su identificación (.....) en mi familia no hay profesionales, mi papá siempre se ha dedicado a la construcción, mi mamá y mi abuela son asesoras de hogar. (Caso 14 que señala ser la primera generación que ingresa a la Universidad

\section{Dimensión 2. Testimonio. Categoría: Experiencias educativas.}

En esta dimensión aparecieron incidentes críticos valorados como relevantes por las emociones que provocaron y la importancia de esto en futuras decisiones que tomaron las entrevistadas en su vida personal y profesional. Las experiencias educativas significativas que influyen en la formación de la IPD y en la decisión de ser docente, tales como: experiencias educativas en cualquier nivel de formación; vivencias con compañeros, profesorado y de clases; descripción de contextos; metodologías utilizadas; prácticas de formación. Se dividen en experiencias positivas y negativas en todos los niveles educativos tal como se puede observar en la Tabla 3 donde se puede advertir lo que corresponde a la dimensión testimonio, sus categorías, subcategorías e incidentes críticos.

Tabla 3: Incidentes críticos de dimensión Testimonio

\begin{tabular}{|c|c|c|}
\hline Categoría & Sub categorías & Incidentes críticos \\
\hline $\begin{array}{l}\text { Experiencias } \\
\text { (antes de ingresar a la } \\
\text { Universidad) }\end{array}$ & $\begin{array}{l}\text { Experiencias educativas } \\
\text { Profesores y características. } \\
\text { Sentimientos provocados. } \\
\text { Experiencias vocacionales }\end{array}$ & $\begin{array}{l}\text { - Experiencias educativas positivas: } \\
\text { Cercanía, acogida, compañeros, } \\
\text { profesorado, clases, contexto, } \\
\text { metodologías utilizadas } \\
\text { - Experiencias educativas Negativas: } \\
\text { Burlas, segregación }\end{array}$ \\
\hline $\begin{array}{l}\text { Experiencias educativas en } \\
\text { formación inicial docente }\end{array}$ & $\begin{array}{l}\text { Experiencias Universidad } \\
\text { Formación inicial docente } \\
\text { Descripción de la Universidad } \\
\text { Descripción de profesores }\end{array}$ & $\begin{array}{l}\text { - Experiencias educativas positivas: } \\
\text { Acogida, afectos, formación, contextos } \\
\text { no convencionales, estímulos, } \\
\text { expectativas. }\end{array}$ \\
\hline & Características de profesores: & -Experiencias educativas negativas: \\
\hline & $\begin{array}{l}\text { afectivas, personales, académicas } \\
\text { Vivencias en la formación. } \\
\text { Sentimientos que le provocan. } \\
\text { Prácticas educativas. } \\
\text { Experiencias vocacionales }\end{array}$ & $\begin{array}{l}\text { Burlas, educación segregada, inducción } \\
\text { a la Universidad, orientación vocacional, } \\
\text { aplicación práctica, preparación del } \\
\text { profesorado de Centros de Práctica }\end{array}$ \\
\hline
\end{tabular}

Respecto a la Categoría de experiencias educativas positivas, antes de ingresar a la Universidad, lo más significativo y valorado es la cercanía, afectos y acogida. Un ejemplo de ello son los colegios cercanos y las relaciones afectivas con el profesorado. En esas instituciones han estudiado sus familiares. Estas experiencias fueron claves para decisiones posteriores que llevan a este profesorado a querer ésta profesión (...) Yo me acuerdo de que era súper cercana a la profesora... su sala era preciosa... y ella estaba sentada al lado tuyo a enseñarte a leer y a escribir. (...) se ponía en la pizarra, cierto, que exponía y bla, bla, blá. (....) hacia súper personalizado el tema de la lectura y escritura, casi en su regazo como una mamá, así que excelente ella. Siempre quise ser como ella (caso 3). 
En la categoría de experiencias educativas negativas antes de ingresar a la Universidad, aparecen como incidentes críticos dos experiencias. La primera, vivencia del profesorado de Educación Especial externa al aula de clases, como docente segregada, y la segunda, la burla que realizaban los compañeros hacia las personas en situación de discapacidad. Otras experiencias educativas negativas era la relación que se establecía entre alumnado y profesorado, a la lejanía y frialdad de los centros formativos y de la individualidad en el comportamiento de cada uno. Señalan que en esta etapa se dieron cuenta la segregación que tenían Ios liceos respecto a la Educación Especial. (.....) igual recibían niños con problemas de aprendizaje y había una bibliotecaria que era psicopedagoga y ella tenía como una especie de doble cargo; y después, en enseñanza media, había una psicopedagoga en el colegio, pero nunca entendí que es lo que hacía, sé que existían, pero nunca vi que hubiera un grupo de integración ni mucho menos (Caso 1).

En la Categoría Experiencias Educativas Positivas, pero en la subcategoría formación inicial docente, (ver Tabla 3), el incidente crítico significativo es un ambiente universitario acogedor, cercano, familiar, había preocupación por la formación integral. La formación cumplió las expectativas de las tituladas, pero señalan, de manera polifónica, que las prácticas en escuelas eran lejanas a la formación recibida en la Universidad y totalmente desconectadas a lo aprendido en ella. Lo estudiado en la Universidad no tenía aplicación en los centros de práctica, esto es el segundo incidente crítico de esta categoría.

Otra experiencia educativa positiva en la formación inicial docente eran las prácticas docentes en contextos no convencionales que dejaron una enriquecedora experiencia. Relatan como una fortaleza las prácticas en liceo de adultos, escuela hospital, colegios rurales y en contextos de vulnerabilidad. (....) el aula hospitalaria fue más en lo personal que en lo profesional, me marcó en lo personal. (Caso 2). En la misma categoría destacaron las experiencias educativas, en la FID, deformadoras. Lo anterior, lo atribuyen, según lo señalado, a la formación del profesorado de los centros de prácticas, la mayoría de ellos tenían formación en otra disciplina, que habían realizado un exiguo perfeccionamiento en Educación Especial, pero trabajaban en esta área. (.....) Por la mala formación, no eran profesoras diferenciales. Eran profesoras de educación básicas o educadoras de párvulos, entonces estaban sacando su mención o ya la habían terminado, no entendían qué pasaba... (Caso 5)

Como categoría emergente en experiencias educativas, en la formación inicial docente, destacaron el proceso de inducción a la Universidad, describiendo el tránsito de la educación secundaria a la Universidad como difícil debido a la complejidad en la exigencia académica, y el temor por no tener claridad del rol que iban a asumir. La edad de ingreso no las acompañaba, eran inmaduras y no estaban preparadas para desafíos. Experimentaron miedo cuando ingresaron, se imaginaban que Educación Especial era para trabajar en una escuela especial, con niños que no iban a poder mejorar. (.....) cuando llegué, ohh en qué me metí... ¿tendré la vocación para hacer esto?, ¿tendré la paciencia para hacer esto? Porque igual me gustaba.(Caso 12)

\section{Dimensión 3. Desarrollo. Categorías: experiencias laborales}

En esta dimensión, como se puede observar en la tabla 4, se destacan como incidentes críticos los siguientes: cambios de trabajo en búsqueda de mejores oportunidades laborales y económicas; la cultura institucional; los despidos; las dificultades para ser aceptadas profesionalmente por profesorado de otras disciplinas y sentirse menoscabadas en su profesión.

Tabla 4: Incidentes críticos de dimensión Desarrollo

\begin{tabular}{|l|l|l|}
\hline Categoría & Subcategorías & Incidentes críticos \\
\hline \multirow{5}{*}{ Experiencias laborales } & $\begin{array}{l}\text { Desempeño laboral } \\
\text { Valoración laboral } \\
\text { Ejercicio profesional } \\
\text { Expectativas laborales } \\
\text { Reforma educativa } \\
\text { Práctica docente }\end{array}$ & $\begin{array}{l}\text { Cultura institucional, cambios de } \\
\text { trabajo, búsqueda de mejores } \\
\text { oportunidades laborales y } \\
\text { económicas, cultura institucional, los } \\
\text { despidos, trabajo burocrático, } \\
\text { desarrollo profesional docente, } \\
\text { bienestar. Discontinuidades. }\end{array}$ \\
\cline { 2 - 3 } & $\begin{array}{l}\text { Penoscipabo profesional, aceptación de } \\
\text { Desempeño laboral } \\
\text { Bienestar docente }\end{array}$ & $\begin{array}{l}\text { Menos. } \\
\text { otros. }\end{array}$ \\
\hline Reformas educativas & $\begin{array}{l}\text { Políticas de educación } \\
\text { especial }\end{array}$ & preparación \\
\hline
\end{tabular}


En la Categoría de experiencias laborales aparecieron como incidentes críticos la presencia de una cultura institucional poco inclusiva, temática pendiente en los lugares de trabajo. La valoración que las entrevistadas hicieron de esto es negativa, ya que aún permanece la cultura de la discapacidad, con un enfoque clínico, segregado cuyas consecuencias discriminan la atención a la diversidad, visto como un favor y no desde la cultura del derecho social. Un segundo incidente crítico, relacionado con el anterior, es un sistema burocrático, muchos antecedentes que completar que disminuye el tiempo de atención al estudiantado, se agrega además, una evaluación psicopedagógica clínica y discriminadora que no siempre es coherente a las prestaciones educativas y a los apoyos. Un tercer incidente crítico, es el acceso al desarrollo profesional docente, esto lo valoran como negativo para ellas y con sentimiento de estancamiento profesional. Solo han podido acceder por iniciativa personal y financiándolos ellas mismas. El perfeccionamiento otorgado por las instituciones donde trabajan es de mala calidad y con escasa aplicación práctica.

En la subcategoría Desempeño Laboral aparecen como incidentes críticos: sentir que fueron preparadas para otro paradigma y que la Universidad, a pesar de saber este cambio, no les advirtió e ingresaron al mundo laboral a desempeñar roles para los que no estaban capacitadas. Opinan que las instituciones donde se desempeñan tampoco están formadas para este cambio, lo que ha provocado que exista tensión en la relación de ellas con los otros profesionales. (.....) me gustaría que las cosas se hicieran mejor y no se puede, es súper difícil, es difícil luchar con las culturas institucionales porque en el colegio particularmente donde yo trabajo, en eso es complejo... esto es lo que hay y así se hace, y hay que trabajar mucho, pero trabajar mucho para objetivos que de repente uno no comparte... (caso 4) (.....) mi experiencia laboral el primer año fue en enseñanza media, es complejo trabajar con profesores de enseñanza media, ellos no están acostumbrados que haya una persona que necesite apoyo en la sala". (Caso 20) (....).el hecho de cambiarse y empezar de nuevo, aparte de conocer a los chicos, conocer a los profesores, a los jóvenes, a los padres y eso es lo más complicado". (Caso 9). Señalan que en momentos de crisis han tenido que ejercer funciones en ambientes laborales para los cuales no tuvieron nada formación, tales como: violencia juvenil; delincuencia; agresiones a los profesores. (....) todavía pasan situaciones, pero ya no como antes, habían... puñaladas, sangre, quemaban los estantes, rompian los vidrios..... (Caso 11).

En la categoría Reforma Educativa, éstas han alterado todo su desarrollo profesional, laboral y personal. El incidente crítico aquí es la sensación de desvalorización profesional, esta situación las han llevado a pensar querer renunciar o cambiar de profesión. Otro problema derivado de la Reforma Educativa es que les ha provocado crisis identitaria, manifestando que no están preparadas para atender todas las Necesidades Educativas Especiales, ya que ellas fueron formadas para atender solo dos de ellas y en las experiencias laborales les corresponde atender a todas. Con las reformas han debido ingresar a aulas donde deben atender a todo el estudiantado, sobre todo a aquellos que presentan necesidades educativas especiales derivadas de discapacidad y no de dificultades de aprendizajes. .... Yo siento que no estoy preparada para atender algunas necesidades. Me he visto enfrentada muchas veces a necesidades que van más allá del aprendizaje ..... (Caso 18)

Relacionado con lo anterior, pero en la subcategoría Bienestar Docente, un incidente crítico es las funciones que deben cumplir en las escuelas teniendo sentimientos de menoscabo laboral. La sensación de ser un suplemento provoca sentimientos de sentirse como el comodín en los ambientes laborales. Las experiencias laborales negativas, ya que se reitera que esto las ha llevado a querer abandonar la profesión, cuestión que en la práctica ninguna de los informantes ha concretado. Expresaron que la valoración de los lugares de trabajo es negativa, se sienten la última profesional de las escuelas. (...) a veces nos tenían de extra, faltaba mucho profesor éramos el comodín. Me tocó hacer ciencia, historia, artes, de todo... yo no estudié para esto. (Caso 12).

\section{Dimensión 4. Final. Identidad Profesional docente}

En esta dimensión, como se observa en la Tabla 5, en la categoría Identidad Profesional docente, según los incidentes críticos analizados, las entrevistadas sienten que han girado a ser asistentes de aula. No quieren despreciar la profesión de éstas profesionales, pero dicen que ellas estudiaron una titulación universitaria de nueve semestres que las prepara para trabajar en pedagogía y ahora, con la nueva política de Educación Especial, el profesorado de aula regular los mira como ayudantes y no como profesionales... pasábamos a ser asistente de aula...vieron que nosotros apoyábamos, entonces vieron la facilidad de pedirnos cosas, entregar las guías, o poder ayudarlos a revisar. Ahora ellos se ven como un trabajo obviamente más. .(Caso 14)

Con respecto al significado que la Identidad Profesional Docente, relatan que tiene como componente 10 social, entendiendo por social como ser un aporte a la sociedad y la transformación de los espacios educativos sobre todo con los sujetos con necesidades educativas especiales. Lo que es contradictorio a lo señalado 
anteriormente, que la Universidad no las preparó para trabajar en estos espacios. ..para mí es poder entregar un aporte a la comunidad escolar, sobre todo a estudiantes que tienen necesidades educativas..... (Caso 1)

Tabla 5: Incidentes críticos de dimensión Final Construcción

\begin{tabular}{|l|l|}
\hline Categoría & Incidentes críticos \\
\hline & $\begin{array}{l}\text { Roles y funciones que deben asumir } \\
\text { (social, emocional, asistentes) }\end{array}$ \\
\hline Identidad profesional docente & Elección profesional \\
\hline & Autoimagen deteriorada \\
\hline & Crisis identitarias \\
\hline
\end{tabular}

En la Tabla 5 se muestran otros incidentes críticos de la categoría Identidad profesional docente, como por ejemplo: las crisis identitarias, señalan estar a gusto con la elección profesional, pero la cantidad de roles que deben asumir, podrían haber estudiado otra cosa. Sería positivo que se les dieran más oportunidades para realizar cursos y proveerse de información con respecto a la cantidad de tareas y responsabilidades que les corresponde realizar. .....lo que pasa es que sigo buscando... después de sacar el diferencial me fui a sacar otro post título, pero ahora quiero aportar más a la educación especial desde de la gestión.....(Caso 15). También hay coincidencia en el aspecto emocional y la IPD, las profesoras se movilizan por aspectos relacionados con lo afectivo como ser psicóloga, mamá, amiga, las que ponen orden y otras características más, pero siempre desde la perspectiva de las emociones. .....Es ser mamá, ser psicóloga, es ser amiga, es ser la mañosa, la verdad, es ser todo, es ser profesora de lenguaje, ser profesora de matemática, ser profesora de historia, de ciencia, es ser por lo menos nosotras, es ser multifacética, tenemos que saber todo y hacer todo. (Caso 19)

\section{DISCUSIÓN}

Con el objeto de dar un orden a la discusión de está investigación, se analizarán los incidentes críticos de acuerdo a un orden temporal o linealidad en el tiempo.

En primer lugar, aparecen incidentes críticos en la categoría identificación donde emerge las construcciones identitarias individuales, la indentización con la Universidad, como una disposición individual, pero al ser generalizada por todas las participantes, es decir colectiva es identificación con la Universidad donde tuvieron la FID, esto es uno de los elementos que aporta de manera importante en la discusión, ya que, es uno de los elementos constituyentes que permite comprender la identidad profesional docente del profesorado. El profesorado principiante señala que ser profesor de Educación Especial es significativo para ellas agregando la mención que estudiaron, destacando una identidad importante con la Universidad.

Lo significativo es que, la literatura plantea que existe vinculación entre identidad, identización e identificación y FID. Existen formas elementales de identificación que configurarían lo esencial de la identidad, por ejemplo: 1) nombres propios; 2) nombres de la función social que cumplen, entre otros. Este incidente crítico tendría un componente importante para la formación de este profesorado, ya que permite reflexionar sobre lo relevante del itinerario formativo de la FID, coincidiendo con Madueño y Márquez (2020), quienes plantean que la formación de pregrado es un componente elemental para la estimulación y refuerzo de posteriores aprendizajes.

En segundo lugar, emergen incidentes crítico en las experiencias educativas anteriores al ingreso a la Universidad, éstas aparecen influenciadas por fondos de identidad (Guitard, 2012) de tipo emocional positivas, tales como: expresión emocional y social, interacciones importantes con personas significativas, como también, de tipo psicológicas. Estos fondos de identidad se constituyen en eventos significativos, es decir incidentes críticos, ya que cuando ocurren reflejan que produjeron importantes modificaciones y decisiones futuras, demostración de ello es, la decisión de ser o no ser profesor. La valoración que se hace de estas experiencias es importante en la construcción de identidad y las decisiones posteriores. Pero, las emociones, no han sido todas positivas, las vivencias negativas abundan, tales como: lejanía del profesorado, las metodologías poco actualizadas, la valoración que realizan las informantes de esto, es no querer ser como ellos.

Lo anterior es importante, ya que, según Cantón y Tardif (2018), la confluencia de la calidad, la satisfacción y la identidad, unido a las expectativas, son los aspectos más definitorios de la identidad que configura a los docentes, aunque también aspectos como el contexto, la formación y la dimensión administrativa ayuden y maticen la identidad construida por los profesores (p.44). El significado de lo anterior es que para cada 
persona la IPD es una experiencia única y su construcción es un fenómeno relacional, dinámico, de acción e interacción con los demás significativos para esa persona (Beijaard et al., 2004). Esta construcción está probablemente definida por el sentido que este profesorado realiza de la labor pedagógica, a partir de las experiencias individuales, que influyen de manera significativa en su desarrollo profesional (Marcelo y Vaillant, 2015).

En tercer lugar, aparecen incidente críticos en la toma de decisión de acceso a la Universidad. Lo que aflora aquí es tener que ingresar, más que poder elegir lo que realmente quieren estudiar. Lo que estimula esta decisión es el desafío de ser profesionales y la meta que constituye para ellos y sobre todo para sus familiares. Esto es coincidente con los resultados de autores como Iglesias y Southwell (2019) quienes concluyen que "los resultados indican que sus familias valoran la formación superior al mismo tiempo que la docencia les ofreció una vía de ascenso social y una salida laboral" (p.464). Las participantes coinciden con esta opinión a pesar de las barreras y obstáculos que sortearon en el itinerario formativo. Señalan que fue mejor que el acceso de sus pares a otras formaciones profesionales (Iglesias y Southwell, 2019). Las dificultades para acceder a la formación universitaria brindan al estudiantado de primera generación posibilidades de tener una experiencia formativa que sus padres no tuvieron, democratizándose así el acceso al saber.

En cuarto lugar, emergen incidentes críticos en las Experiencias educativas en la FID, los resultados permiten comprender que existen vinculaciones significativas positivas entre la formación inicial y la identidad profesional docente. El profesorado principiante ha manifestado satisfacción con la formación inicial recibida en la Universidad, tales como: relaciones cercanas, acogedoras y armoniosas con el profesorado. Esto es coincidente a Van der Wal et al. (2019), quienes plantean en relación a las experiencias positivas o negativas de la práctica docente realizada por los y las docentes participantes en la FID del profesorado principiante, "las tensiones pueden tener un impacto tanto en las prácticas laborales de los docentes como en la identidad profesional de los docentes" (p.69). Esto significa que las experiencias positivas que tiene el profesorado, en este periodo, refuerzan una valoración positiva hacia la formación recibida. Compartimos con que el desarrollo de la identidad profesional docente tiene comienzo en la FID como, aprendizaje por observación, o aprender a enseñar y continúa desarrollándose durante todo el ejercicio profesional (Marcelo y Vaillant, 2015).

En quinto lugar y respecto a la categoría experiencias laborales, aparecen valoradas de manera significativa las decisiones profesionales y las modificaciones de las proyecciones profesionales, como también, la presencia de una cultura institucional poco inclusiva, esto es importante, "ya que autores como González et al. (2019) advierten que al identificar las principales barreras que dificultaban la adecuada atención educativa a la diversidad a nivel general (...) el profesorado ha destacado la falta de formación en atención a la diversidad como una de las principales barreras para funcionar como centros educativos inclusivos (p.258)".

Lo anterior, cultura institucional poco inclusiva, es un tema postergado en los espacios laborales del profesorado, ya que la valoración que las entrevistadas han hecho de esto es negativa, permanece una cultura de la discapacidad, con un enfoque clínico, segregador cuyas consecuencias son que la atención a la diversidad sigue siendo vista como un favor y no desde la cultura del derecho social. Esto ha marcado la trayectoria profesional que han tenido y que las ha estimulado a buscar otras ofertas laborales donde se sientan más identificadas con la cultura para la cual fueron formadas. A pesar de lo anterior, la decisión tomada por todas ellas no llega al extremo de renunciar a la profesión docente, sino permanecer en ella a pesar de todos los obstáculos.

Además, el profesorado principiante ha señalado que los espacios de trabajo son un sistema extremadamente burocrático, coincidiendo en esta opinión con Cantón y Tardif (2018); Marcelo y Vaillant (2015), los resultados de sus investigaciones es que este profesorado tiene mucho trabajo administrativo que realizar y antecedentes que completar que disminuye el tiempo de atención al estudiantado. A lo anterior, las entrevistadas agregaron el nulo acceso al desarrollo profesional docente, y el estancamiento profesional, donde han podido acceder a los perfeccionamientos por iniciativa personal y financiándolos ellas mismas. El perfeccionamiento otorgado por las instituciones donde trabajan es de mala calidad y con escasa aplicación práctica. Pese a lo anterior, la decisión es la búsqueda propia y autofinanciada de desarrollo.

En sexto lugar y en relación al desempeño laboral, emergen resultados que dan cuenta de aspectos que han afectado de manera significativa el desarrollo laboral, son cambios en la política de Educación Especial. El profesorado principiante opinó que las instituciones donde se desempeñan tampoco estaban preparadas para este cambio, lo que ha provocado que exista tensión con otros profesionales. Señalan que en momentos de crisis se han manifestado por tener que ejercer en ambientes laborales para los cuales no tuvieron preparación, tales como violencia juvenil, delincuencia, agresiones a los profesores. Esto ha afectado el nivel de bienestar laboral, ya que el rol que deben cumplir en las escuelas han provocado sentimientos de menoscabo laboral. La sensación de ser un suplemento provoca sentimientos de sentirse como el comodín en los ambientes laborales. 
En séptimo lugar, emergen incidentes críticos, en la categoría IPD, el significado de ser profesor, lo reducen a la búsqueda de recursos, esa es la valorización que realizan de su profesión y de la decisión de permanecer en ella y no renunciar. Plantean que una de las principales acciones que realizan es con relación a buscar alternativas para el aprendizaje de niños con Necesidades Educativas Especiales, para alcanzar lo que se demanda en el sistema educativo. Ser profesor, señalaron, es poder aportar para que el desarrollo del estudiantado con el cual trabajan se realice sin dificultad, con el fin de que aprenda, es decir, situando el foco en esto y no en el aprendizaje (Nichols et al., 2016). En esta misma línea, ser profesor de educación diferencial lo valoran en abordar al estudiantado más allá de lo curricular, en lo familiar, en lo socio cultural, en lo emocional, en la calidad de vida, en el estado de ánimo, la felicidad, nuevamente la centralidad del trabajo y su permanencia en él se sitúa en un plano alejado de el derecho a la educación y la plena participación en ella . Sienten su labor como algo más profundo, genera lazos mucho más grandes con el estudiantado, porque a pesar de que están enfocadas en trabajar con todos, siempre van a tener una conexión con el estudiantado integrado. Sienten que tienen otro tipo de responsabilidad que impide, a pesar de todas las insatisfacciones, a permanecer en esta profesión.

Por último, respecto a la IPD, el profesorado principiante señala que esto tiene que ver con la paciencia, con una conexión emocional con el estudiantado, también en contener y tranquilizar, ser más afectiva, generar lazos positivos, pero con momentos críticos como el cambio permanente de trabajo y tener que empezar todo nuevamente. Según Balderas (2014), el maestro construye su ser docente, de acuerdo a las vivencias que ha tenido a lo largo de su trayectoria personal y profesional, que implican, historia familiar, estudios, formación, ingreso al ámbito laboral y continuidad laboral. Se integra en un entorno laboral específico, existiendo elementos de carácter estructural que lo rodean, por ejemplo, las políticas educativas que definen su desempeño como maestro. Esto significa que en la identidad docente del profesorado hay factores vinculados a sucesos y experiencias vivenciadas de manera personal, intrasubjetiva, social y laboral, intersubjetiva. Es decir, vividos de manera individual y también en relación con los otros. También, esto será variable de acuerdos a los distintos contextos.

\section{DISCUSIÓN FINAL}

El propósito de esta contribución comienza con el planteamiento de un problema poco estudiado en Chile, y que se considera relevante para mejorar los procesos de formación inicial y continua del profesorado en general y de Educación Especial en particular. Los incidentes críticos develaron comportamientos y actitudes significativos en la construcción y/o deterioro de la IPD en la trayectoria de este profesorado. En síntesis, a modo de conclusiones, podemos decir que, a partir de los hallazgos encontrados y el objetivo planteado en este trabajo, la técnica de incidentes críticos, como estrategia de investigación, permite diseñar un patrón explicativo sobre qué sucesos desarrollan la IPD, ya que el análisis de los episodios que han condicionado las trayectorias individuales y profesionales del profesorado principiante de Educación Especial, fundamenta el objetivo de su uso que fue recoger datos sobre la forma en que algunos hechos están relacionados en el desarrollo del objeto epistémico de investigación. Estos pudieron ser investigados e identificados a través del análisis de la interpelación hacia nuevos planteamientos y toma de decisiones respecto a la formación, inicial y continua, de este profesorado. Conforme a lo anterior, para comprender estas trayectorias y replanteamiento vocacional y profesional, se clasificaron los incidentes críticos en una cronología y orden en el tiempo según las dimensiones Inicio, Testimonios, Desarrollo y Final.

El uso de esta técnica se hace indispensable para analizar qué factores están vinculados en la IPD. Utilizarla en la investigación educativa es sugerible, tanto en la formación inicial del profesorado, como también en educación continua y en estudios de postgrado. Finalmente, podemos decir que se ha podido perfilar una serie de incidentes críticos que ha posibilitado comprender qué sucesos han ido construyendo la IPD y entender las decisiones que han tomado, las identidades desarrolladas y las razones que han tenido para esto. Sería interesante, realizar estudios longitudinales con este profesorado, ya que la identificación y análisis basado en Incidentes Críticos supone un área de investigación e intervención que puede resultar realmente fructífera al abrir un espacio entre las teoría, concepciones, creencias y sentimientos que sostienen los docentes y que determinan, en buena parte, sus decisiones y conductas. Esto permitiría conocer qué modifica la identidad profesional docente del profesorado principiante desde su inicio hasta llegar a ser profesorado experto. Un análisis de este tipo reflejaría la vinculación que tienen las experiencias laborales, personales y sociales en esta etapa del desarrollo de la IPD.

Finalmente, es importante estimular las posibilidades que tiene la técnica del incidente crítico como herramienta metodológica para la investigación (Gremler, 2004) y la formación del profesorado de educación especial, así como también, evaluar el impacto que esta técnica tiene en la comprensión y desarrollo de la identidad docente y toma de conciencia acerca de qué desarrolla la identidad docente de este profesional 
según las decisiones tomadas y transformaciones personales y profesionales que se han producido en sus trayectorias docentes.

\section{CONCLUSIONES}

En concordancia a los resultados de esta investigación y de su discusión, de la comparación con resultados de otros autores y del cuidadoso análisis expuesto se pueden obtener las siguiente conclusiones principales:

1.- El análisis de los incidentes críticos permite describir episodios que condicionan las trayectorias individuales y profesionales del docente principiante de Educación Especial, de esta manera logran caracterizar valoraciones de eventos de vida o situaciones críticas que han estado relacionados con decisiones profesionales y personales del profesorado.

2.- Estos momentos críticos representan situaciones imprevistas en las que el profesorado se siente llamado a actuar, ya sea tomando otros rumbos o intentando resolverlos de alguna manera. Éstos, considerados como incidentes y experiencias significativas, revelan prototipos de identidad docente profesional, que se pueden situar en la adaptación forzada, en una identidad que responde a lo normado, en una identidad frustrada, pero finalmente aceptada.

3.- Los elementos que desarrollan la identidad docente son experiencias educativas positivas o negativas. Éstas serían claves en situaciones inesperadas. Otros elementos serían el sentimiento de desvinculación existente entre formación teórica y práctica en la FID. Así como, en el ejercicio laboral, aparece el sentimiento de menoscabo, la baja autoestima, expectativas truncadas y la persistencia de culturas institucionales poco inclusivas, desarticuladas y excluyentes.

4.- Las instituciones que tienen responsabilidades en la formación del profesorado, ya sea inicial o continua, necesitan integrar en los itinerarios formativos, procesos que estimulen y promuevan, de manera explícita, la identidad docente, esto porque quien se apropia del ser profesorado tendrá mayor implicancia en su significado y disposición para desempeñarse con facultad en el ejercicio profesional.

5.- Por último, es necesario atender que la inserción o inducción a la docencia no es un recorrido sencillo de llevar a cabo, y este se realiza en vinculación a otros, en espacios con particularidades y complejidades únicas, con cultura propias y situaciones educativas heterogéneas. De esta manera, para sortear muchas complejidades que se presentan, es recomendable procesos de inducción laboral sistematizados y evaluados de manera permanente para evitar todo lo relacionado con el impacto que produce lo aprendido y la realidad que produce tantas insatisfacciones y frustraciones.

\section{REFERENCIAS}

Albores, I., y Avendaño, V., La identidad docente de los profesores del Colegio de Bachilleres de Chiapas, Revista Criterios, ISSN: 0121-8670, 23 (1), 47-58 (2016)

Avalos B., Learning from Research on Beginning Teachers. In: Loughran J., Hamilton M. (eds), https://doi.org/10.1007/978-981-10-0366-0_13, International Handbook of Teacher Education, Springer, Singapore (2016)

Beijaard, D., Meijer, P. C., y Verloop, N., Reconsidering research on teachers' professional identity: http://doi.org/10.1016/j.tate.2003.07.001, Teaching and Teacher Education, 20 (2), 107-128 (2004)

Balderas, I., Propuesta de guión de entrevista para el estudio de la identidad docente. Revista Latinoamericana de Metodología de la Investigación Social, ISSN 1853-6190, 6, (3), 73-87 (2014)

Bolívar, A., Domingo, J. y Fernández, M., La investigación biográfico narrativa en educación.: La Muralla, S.A. (2001)

Buchanan, R., Teacher identity and agency in an era of accountability: https://doi.org/10.1080/13540602.2015.1044329, Teacher and Teaching, 21(6), 700-719 (2015)

Bukor, E., Exploring teacher identity from a holistic perspective: Reconstructing and reconnecting personal and professional selves: https://doi.org/10.1080/13540602.2014.953818, Teachers and teaching, 21(3), 305-327 (2015)

Cantón, I., y Tardif, M., Identidad profesional docente. Narcea: Ediciones (2018)

Colliander, H., The experienced newcomer - The (Trans) forming of professional teacher identity in a new landscape of practices: https://doi.org/10.1016/j.tate.2017.10.012, Teaching and Teacher Education, 69, 168-176 (2018)

Correa, J. M., Martínez-Arbelaiz, A., y Gutiérrez, L. P., Between the real school and the ideal school: another step in building a teaching identity: https://doi.org/10.1080/00131911.2013.800956, Educational Review, 66 (4), 447-464 (2014) 
Deng, L., Zhu, G.,y otros cuatro autores, Emociones, dilemas y formación de la identidad profesional de los futuros docentes en medio de las prácticas docentes, https://doi.org/10.1007/s40299-018-0404-3, The Asia-Pacific Education Researcher, 27 (6), 441-453 (2018)

Gremler, D. D., The critical incident technique in service research: https://doi.org/10.1177/1094670504266138, Journal of Service Research, 7(1), 65-89 (2004)

González-Gil, F., Martín-Pastor, E., y Castro, R. P., Educación inclusiva: barreras y facilitadores para su desarrollo. Un estudio desde la percepción del profesorado, doi: https://doi.org/10.30827/profesorado.v23i1.9153, Revista de Currículum y Formación del Profesorado, 23(1), 243-263 (2019)

Guitart, M., La multimetodología autobiográfica extendida (MAE). Una estrategia cualitativa para estudiar la identidad, los fondos de conocimiento y las formas de vida: https://www.redalyc.org/articulo.oa?id=28025469002, Revista Electrónica de Metodología Aplicada, 17(2), 51-64 (2012)

Iglesias, A., y Southwell, M. Paradojas de formarse como docente siendo "recién llegados": http://dx.doi.org/10.7213/1981-416X.19.060.AO07, Revista Diálogo Educacional, 19 (60), 447-468 (2019)

Jara, C., y Mayor-Ruiz, C., Explorar la Construcción de la identidad docente en profesionales de la salud: diseño y validación de instrumento: http://dx.doi.org/10.4067/S0718-50062019000100013, Formación universitaria, 12(1), 13-24 (2019)

Madueño, M. L., y Márquez, L., Formación de la identidad docente de estudiantes de la carrera de Educación Primaria desde la experiencia de la práctica profesional: doi: https://doi.org/10.6018/j/333091, Formación universitaria, 13(5), 57$68(2020)$

Marcelo, C., y Vaillant, D., El A, B, C y D, de formación docente.: Narcea (2015)

Marcelo, C., Burgos, D., y otros cinco autores, La inducción del profesorado principiante en la República Dominicana. El programa INDUCTIO: https://doi:10.6018/red/48/4, Revista Iberoamericana de Educación, 71(2), 145-168 (2016)

Ministerio de Educación de Chile. Estándares orientadores para carreras de educación especial.: MINEDUC. (2013)

Mulone, M. El proceso de construcción de la identidad profesional de los traductores de inglés:

https://doi.org/10.22201/iisue.20072872e.2016.19.1102, Revista Iberoamericana de Educación Superior, 7(19) (2016)

Nichols, S. L., Schutz, P. A., Rodgers, K., y Bilica, K., Early career teachers' emotion and emerging teacher identities: https://doi.org/10.1080/13540602.2016.1211099, Teachers and Teaching, 23 (4), 406-421 (2016)

Noonan, J., An Affinity for Learning: Teacher Identity and Powerful Professional Development: https://doi.org/10.1177/0022487118788838, Journal of Teacher Education, 70(5), 526-537 (2019)

Pappa, S., Moate, J., Ruohotie-Lyhty, M., y Eteläpelto, A., Teachers' pedagogical and relational identity negotiation in the Finnish CLIL context: https://doi.org/10.1016/j.tate.2017.03.008 , Teaching and Teacher Education, 65, 61-70 (2017)

Pillen, M. T., Den Brok, P. J., y Beijaard, D., Profiles and change in beginning teachers' professional identity tensions: https://doi.org/10.1016/j.tate.2013.04.003, Teaching and Teacher Education, 34, 86-97 (2013)

Pujadas, J., El método biográfico: el uso de las historias de vida en ciencias sociales. Madrid, España: Centro de Investigaciones Sociológicas (2002)

Rodgers, C. R., y Scott, K. H., The development of the personal self and professional identity in learning to teach. En autores Handbook of research on teacher education, Routledge, New York (2008), pp. $732-755$ (2008)

Vaillant, D., y Cardozo-Gaibisso, L., Desarrollo profesional docente: entre la proliferación conceptual y la escasa incidencia en la práctica de aula: doi: https://doi.org/10.29197/cpu.v13i26.259, Cuadernos de Pedagogía Universitaria, 13 (26), 5-14 (2016)

Van der Wal, M. M., Oolbekkink-Marchand, H. W., Schaap, H., y Meijer, P. C., Impact of early career teachers' professional identity tensions: https://doi.org/10.1016/j.tate.2019.01.001, Teaching and Teacher Education, 80, 59-70 (2019)

Vloet, K., Klatter, E., Janssen, S., y Kessels, G. Bumpy moments considered as critical incidents in dialogue: professional Identity of technical VET-teachers: https://doi: 10.5565/rev/qpsicologia.1573, Quaderns de psicologia, 22(2), 009 (2020) 